\title{
Débitmétrie d'un écoulement diphasique de propane
}

\author{
E. Hervieu (1)
}

Commissariat à l'Energie Atomique

La maîtrise des «Risques Technologiques Majeurs " liés au stockage des Gaz de Pétrole Liquéfiés (GPL) suscite depuis quelques années de nombreuses études de sûreté, dont l'objectif final concerne la possibilité de déterminer l'étendue du périmètre de sécurité autour d'une installation de stockage. En effet, il s'avère que les résultats d'une Analyse Quantitative de Risques (AQR) peuvent être entachés d'une bande d'incertitude très large en raison du caractère grossier des modèles employés et de leur manque de validation expérimentale. L'étude présente s'intègre donc dans un effort de recherche sur les mécanismes physiques impliqués dans les scénarios d'accident concernant les systèmes de stockage de gaz liquéfié inflammable.

$\mathrm{Si}$ on détaille, lors d'une AQR, le scénario d'un accident causé par une fuite, la quantification des effets d'une explosion et l'évaluation des dommages qui en résultent requièrent la connaissance du «Terme Source», qui désigne l'ensemble des conditions précédant la dispersion des produits dans l'atmosphère, c'est-à-dire principalement la nature physique de l'effluent à la brèche ainsi que l'évolution temporelle de son débit.

\section{L'installation expérimentale}

Afin d'étudier les effets de la décompression d'un réservoir sur l'écoulement à la brèche, une installation expérimentale à échelle réduite a été conçue $[1,2,3,4]$. L’impossibilité d'extrapoler des résultats qui seraient obtenus avec un fluide de substitution nous a conduit à utiliser le fluide d'intérêt réel, c'est-à-dire le propane.

L'installation (fig. I) est composée d'un réservoir de décompression $\left(9 \mathrm{dm}^{3}\right)$ relié à un réservoir d'expansion $\left(2,5 \mathrm{~m}^{3}\right)$ par une ligne de décharge de $12 \mathrm{~mm}$ de diamètre

(') Centre d'Etudes Nucléaires de Grenoble. Service de Thermohydraulique pour les Applications Industrielles, 17, rue des Martyrs, 38054 Grenoble cedex 9. Tél. : 76884533 . Fax : 76885036 . intérieur. Cette conduite comporte une vanne à ouverture rapide, et son extrémité inférieure reçoit un orifice calibré interchangeable qui permet de faire varier la taille de la brèche étudiée.

Le réservoir de décompression est destiné à contenir le propane liquide dans des conditions de saturation. Il est muni de capteurs de pression, de thermocouples immergés, de thermocouples de paroi et d'une sonde de niveau. Il peut être refroidi ou chauffé entre 0 et $+60^{\circ} \mathrm{C}$ afin de faire varier la pression interne entre 5 et 18 bar.

Le réservoir d'expansion, instrumenté en pression et température, est destiné à recevoir la charge de propane en phase gazeuse au cours de la vidange du réservoir de décompression. Ce propane est brûlé en fin d'essai.

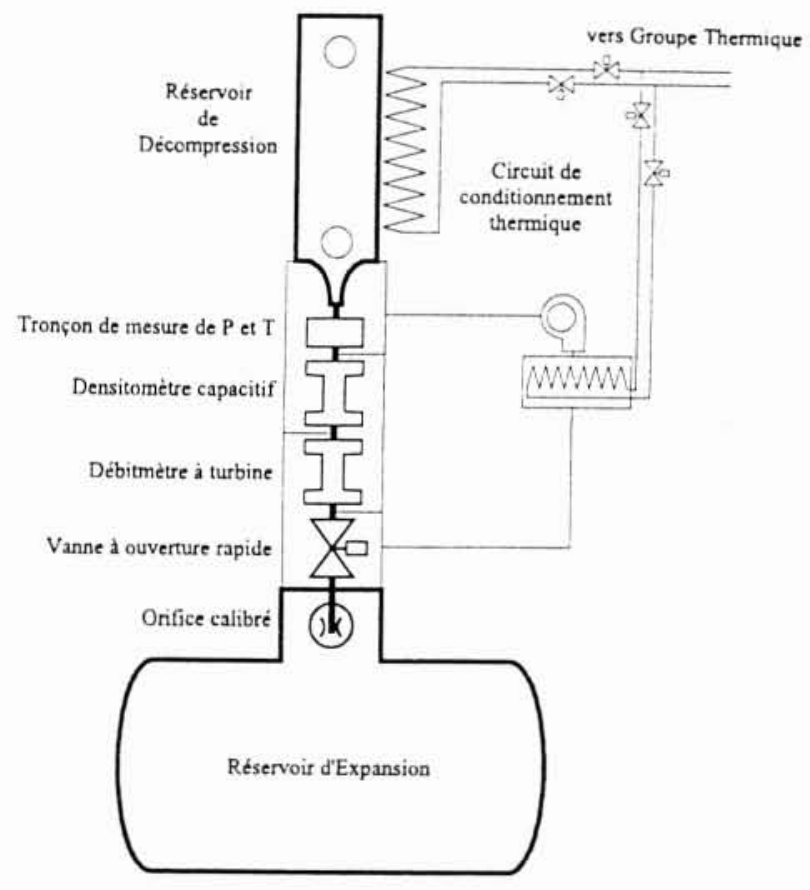

1. L'installation expérimentale GPL.

Flow measurement of a propane two-phase flow

In order to study the decompression of a propane storage tank, a small-scale facility has been constructed. During the decompression, the flow may be single-phase liquid, two-phase and then single-phase vapor. The mass flowrate is measured with a combination of two sensors : a turbine volumetric flowmeter and a capacitive impedance densitometer which measures the void fraction in the two-phase mixture. 


\section{Principe de débitmétrie}

Au cours de la vidange du réservoir, l'écoulement de propane peut être successivement monophasique liquide, diphasique puis monophasique gazeux.

Le comportement de moulinets en écoulement diphasique a été modélisé par POPPER [5, 6] puis par ROUHANI [7], qui proposent des formulations pratiquement équivalentes. A partir du bilan de quantité de mouvement angulaire entre le fluide et les pales et en tenant compte des vitesses phasiques distinctes, ROUHANI montre que la fréquence $f$ de rotation du rotor peut s'exprimer sous la forme suivante :

$$
f=\lambda_{2 \phi} \dot{M}\left(\frac{x^{2}}{\alpha_{M} \rho_{L}}+\frac{(1-x)^{2}}{\left(1-\alpha_{M}\right) \rho_{G}}\right)
$$

où $\dot{M}$ est le débit massique, $x$ le titre massique, $\alpha_{M}$ le taux de vide dans le moulinet, $\rho_{L}$ et $\rho_{G}$ les masses volumiques des phases liquide et gazeuse.

FRANK et al. [8] ont montré que l'on pouvait utiliser le modèle de RoUHANI en prenant comme coefficient de débit diphasique $\lambda_{2 \Phi}$ la valeur délivrée par un étalonnage réalisé en écoulement monophasique liquide.

La détermination du débit massique nécessite donc la mesure des masses volumiques phasiques, de la fréquence de rotation du rotor, du taux de vide et du titre massique. En l'absence de ce dernier, nous en sommes réduits à utiliser l'hypothèse que l'écoulement est homogène dans le moulinet.

Ainsi :

$$
\dot{M}=\left(\alpha \rho_{G}+(1-\alpha) \rho_{L}\right) \frac{f}{\lambda_{2 \phi}}
$$

En conséquence, sur l'installation expérimentale que nous décrivons, la ligne de décharge est munie de trois manchettes instrumentées. La première est destinée à la détermination des masses volumiques phasiques. La seconde est un densitomètre permettant de mesurer le taux de vide. La troisième manchette est un moulinet.

\section{Mesure des masses volumiques phasiques}

Les masses volumiques $\rho_{L}$ du liquide saturé et $\rho_{G}$ de la vapeur saturée sont déduites de la pression et de la température mesurées dans la ligne de décharge.

Pour ce faire, une manchette comporte un thermocouple de type $K$ dont la soudure chaude est soudée à la gaine. Cette dernière, de $0,25 \mathrm{~mm}$ de diamètre, pénètre d'environ $3 \mathrm{~mm}$ dans l'écoulement au travers d'un trou de $1 \mathrm{~mm}$ de diamètre (fig. 2). Les données fournies par le thermocouple sont traitées avec les tables internationales de f.e.m. en fonction de la température. La précision escomptée est de

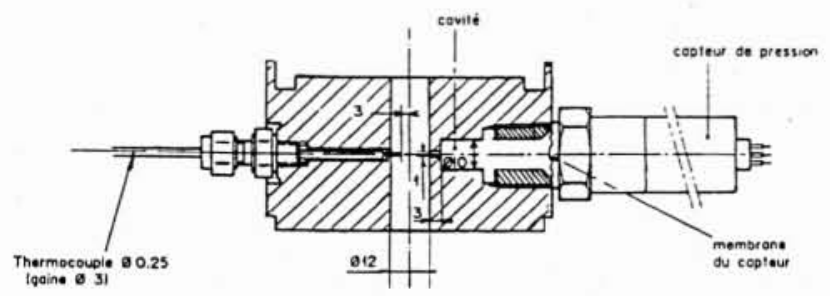

2. Manchette de mesure de pression et température. $\pm 1^{\circ} \mathrm{C}$. Le temps de réponse de ce thermocouple a été estimé inférieur à $10 \mathrm{~ms}$ pour une vitesse d'écoulement de $10 \mathrm{~m} / \mathrm{s}$.

La manchette comporte également un capteur piézorésistif KELLER permettant de mesurer la pression dans une gamme de 0-20 bar absolus, avec une précision intrinsèque (linéarité + hystérésis + répétabilité) de $\pm 0,2 \%$ de la pleine échelle. L'influence de la température est de $0,003 \%$ de la pleine échelle par ${ }^{\circ} \mathrm{C}$. Les performances annoncées laissent donc prévoir une précision globale de $\pm 0,08$ bar pour un fonctionnement entre 0 et $60^{\circ} \mathrm{C}$. Le temps de réponse de ce capteur est inférieur à $0,1 \mathrm{~ms}$.

\section{Mesure du débit volumique}

\subsection{Le moulinet et la détection}

Le moulinet a été conçu au Centre d'Etudes Nucléaires de Grenoble (CEA), par le Groupe Technologie et Instrumentation du Service de Thermohydraulique des Réacteurs (STR) et réalisé par le Laboratoire d'Etudes Thermohydrauliques de Systèmes, du même service.

Il comporte (fig. 3) un équipage amovible constitué d'un stator et d'un rotor, qui s'insère en butée dans la conduite de diamètre $12 \mathrm{~mm}$, elle-même percée dans le corps en inox de la manchette de longueur $150 \mathrm{~mm}$. L'équipage est maintenu en place par une entretoise, elle aussi de diamètre interne de $12 \mathrm{~mm}$. Le rotor, usiné dans la masse par électroérosion, comporte 12 pales de profil plat au pas de $120 \mathrm{~mm}$. Le volume écoulé par tour, calculé par des considérations géométriques, est de $10,454 \mathrm{~cm}^{3}$.

Conçu pour les essais à haute pression et à haute température, le rotor est monté sur deux roulements à billes non lubrifiés. Les deux extrémités de l'axe sont convexes et viennent s'appuyer sur deux butées planes du stator en carbure de tungstène, de façon à réduire le frottement mécanique et à compenser les efforts axiaux subis par le rotor. Ce système permet au rotor d'accepter des vitesses de rotation élevées (20000 tours/s) mais surtout de subir de fortes accélérations $\left(600000\right.$ tours $\left./ \mathrm{s}^{2}\right)$, ce qui le rend particulièrement bien adapté aux écoulements liquidevapeur.

Le système de détection du passage des pales est une bobine à noyau aimanté, type Faure-Hermann, insérée dans un logement cylindrique du corps perpendiculaire à l'axe, au niveau des pales du rotor. Le signal issu de ce détecteur

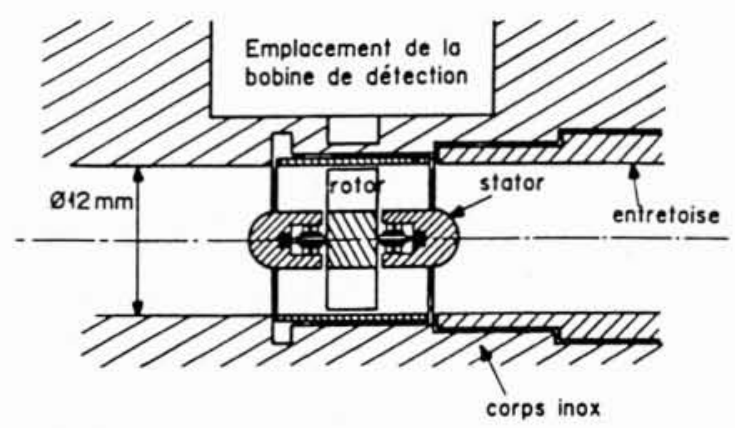

3. Equipage amovible du moulinet. 
magnétique est traité par deux fréquencemètres numériques dont les gammes respectives sont $[50-1000 \mathrm{~Hz}]$ et [500-10 $000 \mathrm{~Hz}]$. On évite les mesures à fréquence inférieure à $50 \mathrm{~Hz}$, où le couple résistant engendré par le détecteur magnétique l'emporte sur les forces hydrodynamiques.

\subsection{L'étalonnage}

Le banc d'étalonnage a été conçu par le Laboratoire d'Etudes Thermohydrauliques de Systèmes (STR/CENG/CEA). Il permet d'étalonner, en eau froide $\left(20^{\circ} \mathrm{C}\right)$ et chaude $\left(80^{\circ} \mathrm{C}\right)$, des débitmètres dans une plage de 0,1 à $200 \mathrm{~m}^{3} / \mathrm{h}$.

La mesure de référence du débit massique est effectuée par pesée continue de la masse d'eau s'écoulant dans une cuve située à l'aval du débitmètre. La régularité du débit est assurée par une cuve à niveau constant qui fournit une charge de $10 \mathrm{~m}$ avec des fluctuations de $\pm 2 \mathrm{~cm}$. L'incertitude relative sur le débit massique pour toute la gamme de fonctionnement du banc d'étalonnage est inférieure à $310^{-3}$. Le débit volumique $Q_{\text {ref }}$ est déduit du débit massique à l'aide des tables de la masse volumique de l'eau. Compte tenu de l'incertitude absolue sur la mesure de température de l'eau qui est de $\pm 0,5^{\circ} \mathrm{C}$, l'incertitude relative sur le débit volumique $Q_{\text {ref }}$ est inférieure à $3,510^{-3}$.

L'incertitude sur la mesure de fréquence $f$ de passage des pales est évaluée de façon statistique. Le temps écoulé pendant le passage de 100 pales est compté (avec une incertitude relative inférieure à $510^{-5}$ ), puis inversé pour estimer $f$. Pour chaque point d'étalonnage, cette mesure est répétée au moins 100 fois. En supposant que ces mesures se répartissent suivant une loi normale, la moyenne et l'écart type de chaque série permettent d'estimer l'incertitude sur la détermination de $f$. Sur la plage couverte par les campagnes d'étalonnage effectuées, l'incertitude relative sur la mesure de fréquence $f$ s'est toujours avérée inférieure à $10^{-3}$.

La figure 4 présente les points d'étalonnage du moulinet. Suivant la méthode de corrélation de HOCHREITER [9], nous présentons l'évolution du coefficient de débit $C$ en fonction du nombre de Reynolds $\mathrm{R}_{e}$, lesquels sont définis par:

$$
\begin{aligned}
& R_{e} \triangleq 2 \pi \frac{d^{2}}{12} \frac{f}{v} \\
& C \triangleq \frac{12}{2 \pi d^{3}} \frac{Q_{\mathrm{ref}}}{f} .
\end{aligned}
$$

Les valeurs du coefficient de débit sont à comparer, sachant que le moulinet engendre un volume de $10,454 \mathrm{~cm}^{3} /$ tour, à la valeur théorique de 0,963 .

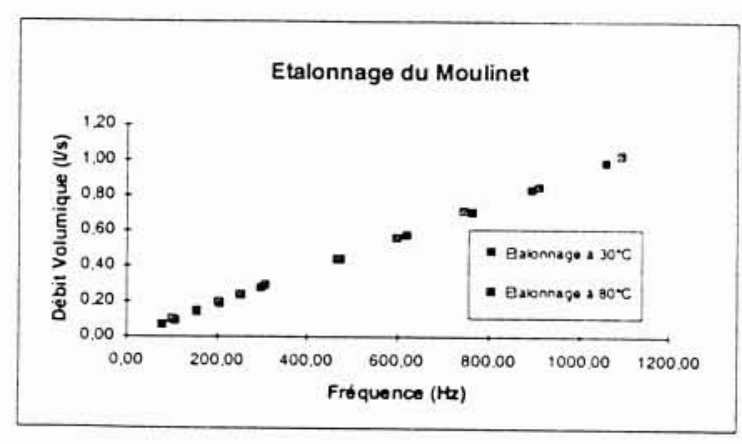

4. Courbes d'étalonnage du débitmètre à turbine.
A température égale, le propane a une viscosité cinématique nettement inférieure à celle de l'eau. A $20^{\circ} \mathrm{C}$, $v_{\text {eau }} / v_{\text {propane }} \approx 5$. L'étalonnage en eau à $80^{\circ} \mathrm{C}$ ramène ce rapport à une valeur plus acceptable, puisque $v_{\text {cau }}$ $\left(80^{\circ} \mathrm{C}\right) / v_{\text {propanc }}\left(20^{\circ} \mathrm{C}\right) \approx 1,7$. En pratique, nous utiliserons donc les résultats de l'étalonnage à $80^{\circ} \mathrm{C}$.

\section{Mesure du taux de vide}

Parmi les différentes techniques répertoriées de mesure du taux de présence volumique de la phase gazeuse dans un écoulement liquide-gaz ou liquide-vapeur, la méthode électrique a été retenue. Basée sur la mesure de l'impédance du milieu diphasique, elle nous a paru la mieux adaptée aux conditions particulières de notre installation pour les raisons suivantes :

- faible temps de réponse, indispensable compte tenu de l'instationnarité de l'écoulement,

- non-intrusivité du capteur dans l'écoulement,

- alimentation électrique de faible puissance, donc fonctionnement en sécurité intrinsèque vis-à-vis du propane.

La cellule de mesure et l'électronique de traitement qui lui est associée sont d'une conception originale, assurée par le Laboratoire d'Etudes Fondamentales du Service de Thermohydraulique pour les Applications Industrielles (STI/CENG/CEA).

\subsection{Principe de mesure}

Le propane liquide est un isolant électrique, ce qui implique de réaliser une mesure d'impédance capacitive (par opposition à une mesure d'impédance résistive qui peut être mise en œuvre avec des fluides conducteurs). Le densitomètre capacitif mesure les variations de permittivité diélectrique du mélange diphasique induites par les variations de taux de vide. La permittivité diélectrique $\varepsilon_{L}$ du liquide décroît de 1,6 à 1,4 entre 10 et $+60^{\circ} \mathrm{C}$, tandis que celle $\varepsilon_{G}$ de la vapeur reste constante à une valeur proche de 1 . Le manque de contraste entre les phases que dénote le faible rapport $\varepsilon_{L} / \varepsilon_{G}(1,6$ pour le propane, il vaut 80 pour le couple eau-air !) engendre une faible sensibilité de la mesure envers le taux de vide. En revanche, cette absence de contraste favorise l'indépendance de la mesure capacitive vis-à-vis de la configuration des phases.

\subsection{Description du capteur}

La méthode capacitive réserve la possibilité de placer les électrodes en retrait derrière un isolant, et ainsi de suppri-

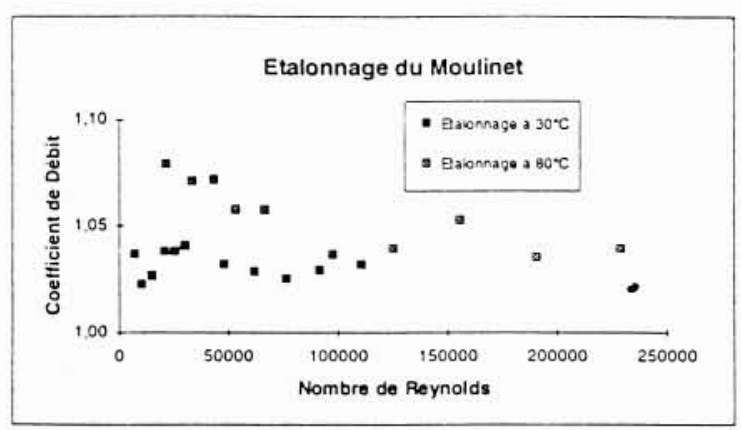


mer tout contact électrique direct avec le propane. Cet avantage sur le plan de la sûreté a été exploité lors de la conception du capteur. Ainsi, l'âme centrale de l'appareil est un barreau en Téflon (fig. $5 a$ ) de section rectangulaire, percé dans l'axe au diamètre hydraulique de la ligne de décharge $(12 \mathrm{~mm})$ et dans lequel circule le mélange diphasique. Sur ce barreau sont collés trois types d'électrodes :

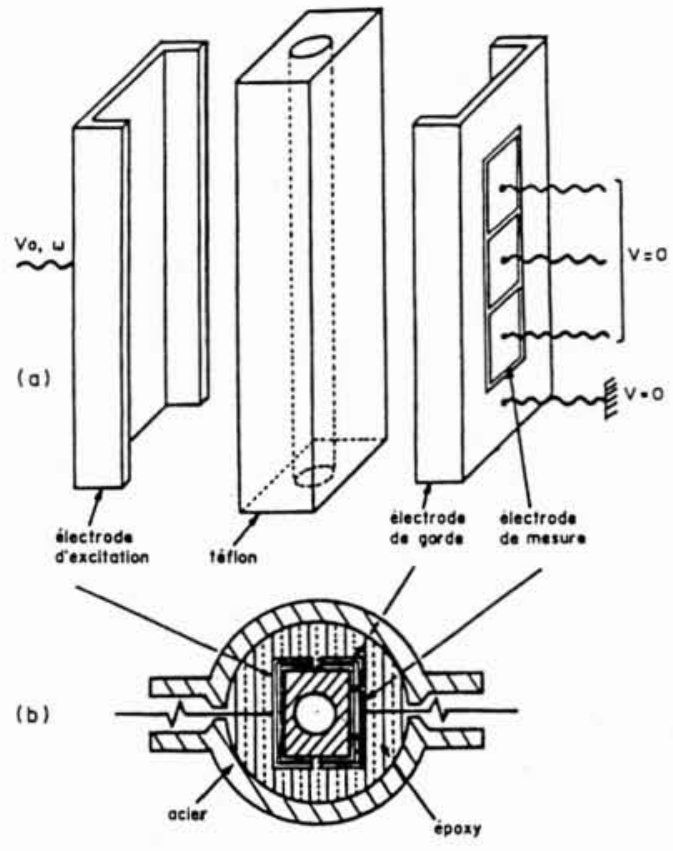

5. Densitomètre capacitif.

- une électrode d'excitation de section en $U$ portée à un potentiel alternatif de fréquence $20 \mathrm{kHz}$ et de tension de crête de quelques volts,

- trois petites électrodes de mesure planes et rectangulaires placées côte à côte suivant l'axe de la conduite, qui servent à mesurer de façon indépendante les courants transmis par l'électrode d'excitation à travers l'épaisseur de Téflon et le mélange diphasique (chacune étant connectée à l'entrée d'un convertisseur courant-tension, à un potentiel très proche de la masse),

- une électrode de garde en $U$, évidée à l'emplacement des électrodes de mesure (reliée à la masse, elle a pour fonction de redresser les lignes de champs sur le bord des électrodes de mesure de telle sorte que le volume de mesure soit bien défini).

L'ensemble barreau en Téflon-électrodes collées ne résisterait pas à une pression interne pouvant atteindre 18 bar. Il est donc inséré dans une coque cylindrique en acier, équipée de brides. Entre la coque et le barreau, l'espace annulaire est rempli de résine époxy (fig. $5 b$ ). Outre sa fonction mécanique, la coque d'acier est connectée à la masse, et joue donc le rôle de blindage électrique pour les électrodes.

\subsection{Critères de conception}

La figure $6 a$ représente, sur une coupe transversale du bloc de mesure, le schéma électrique équivalent du capteur capacitif. La capacité à mesurer $C_{m}$ apparaît entre l'électrode d'excitation et l'électrode de mesure maintenue au potentiel $0 \mathrm{~V}$ par le système de mesure, lequel est représenté ici par un ampèremètre. L'électrode de mesure reçoit un courant principal affecté par toutes les zones du fluide, correspondant aux lignes de champ traversant la conduite, et un courant secondaire dû aux lignes de champ contournant la conduite (fig. 6 b). Le courant principal traverse le milieu diphasique et une certaine épaisseur d'isolant, ce que l'on représente par deux capacités $C_{s}$ en série avec la capacité $C_{F}$ de la zone fluide; le courant secondaire s'ajoute au courant principal, ce que l'on représente par deux capacités $C_{p}$ en parallèle entre les électrodes. La capacité équivalente $C_{m}$ est donnée par:

$$
C_{m}=2 C_{p}+\frac{C_{F}(\alpha)}{1+2 \frac{C_{F}(\alpha)}{C_{s}}} .
$$

On a écrit ici $C_{F}(\alpha)$ pour montrer la capacité importante pour la mesure, mais il faut noter que $C_{p}$ et $C_{s}$ ne sont pas indifférentes à la permittivité du mélange dans la conduite, donc au taux de vide $\alpha$.

La géométrie particulière du densitomètre nécessite un choix judicieux de l'isolant (permittivité relative $\varepsilon_{s}$ ) et des trois paramètres dimensionnels des électrodes (fig. $6 c$ ). Les contraintes de cette optimisation se situent à différents niveaux :

- comportement électrique global (importance relative des différentes capacités de l'équation 5),

- comportement électrique local (homogénéité du champ électrique dans la conduite, afin de minimiser l'influence de la configuration des phases de l'écoulement sur la mesure),

- sensibilité à la température,

- intégrité mécanique de l'assemblage.

L'examen des lignes de champ, obtenues par résolution de l'équation de LAPLACE dans une géométrie et avec des conditions aux limites représentatives, peut apporter des informations intéressantes pour le choix de ces paramètres. La géométrie ne se prête pas à un calcul analytique du potentiel, aussi avons-nous effectué la résolution par une méthode numérique d'éléments finis.

On a choisi un isolant de faible permittivité, de valeur proche des permittivités des deux phases de l'écoulement, de façon à limiter la tendance des lignes de champ à contourner la conduite. On rend ainsi le champ plus homogène dans la zone de l'écoulement, ce qui garantit une bonne indépendance vis-à-vis de la configuration des phases. Par ailleurs, l'isolant doit être de permittivité peu sensible à la température, sous peine de rendre la mesure de capacité dépendante de la température du densitomètre. Le choix du Téflon comme isolant a été motivé par ces considérations $\left(\varepsilon_{s}=2,1, d \varepsilon_{s} / d T=4,310^{-4}{ }^{\circ} \mathrm{C}^{-1}\right)$.

L'étude numérique de l'influence des caractéristiques géométriques de ce capteur nous a conduit à adopter une section d'isolant de $24 \mathrm{~mm} \times 18 \mathrm{~mm}$ (fig. $6 c: a_{i}, b_{j}$ ) et une largeur d'électrode de mesure de $12 \mathrm{~mm}$ (fig. $6 c: a_{m}$ ).

Dans la direction axiale, une longueur d'électrode de $20 \mathrm{~mm}$ a été choisie, pour répondre à des considérations d'encombrement et de sensibilité de mesure. En effet, compte tenu de ces dimensions, des caractéristiques diélectriques du propane et du Téflon, et de la fréquence d'excitation, le contraste capacitif $C_{m}(\alpha=0)-C_{m}(\alpha=1)$ 
(a)

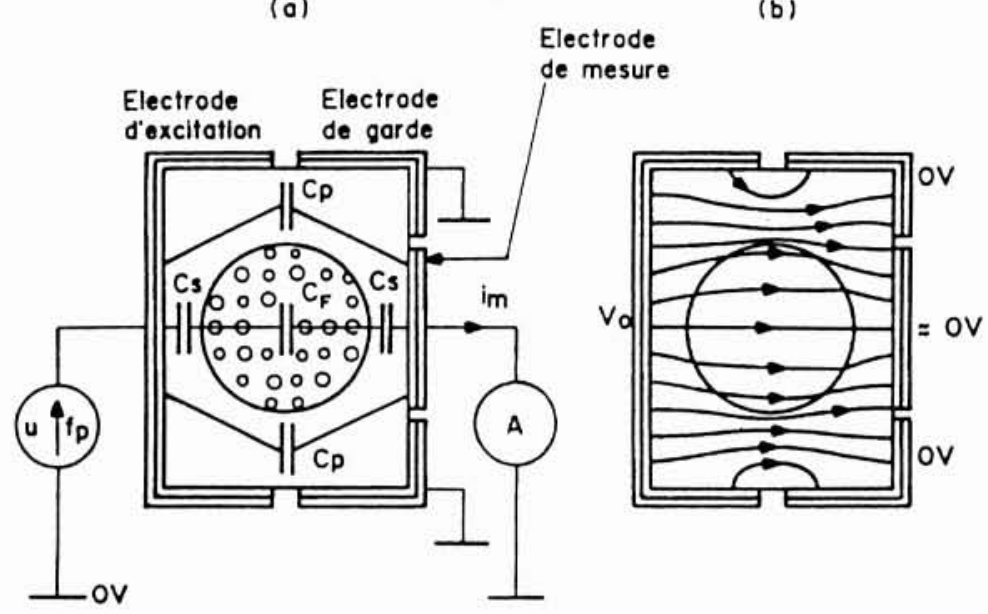

(c)

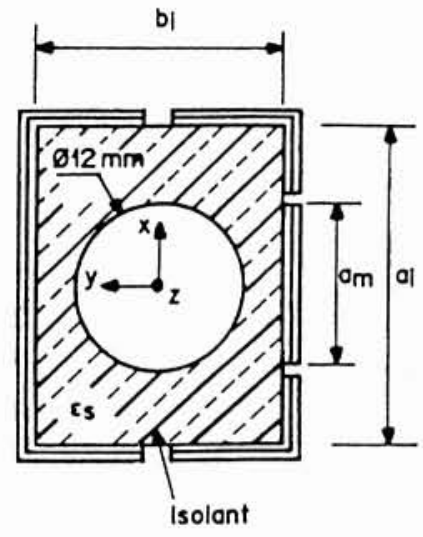

6. Densitomètre capacitif. Schéma électrique équivalent (a), lignes de courant (b), paramètres à optimiser (c).

est de $0,1 \mathrm{pF}$, autour d'une valeur moyenne du même ordre de grandeur. Cela signifie que le courant qui traverse le dispositif est de l'ordre de $0,1 \mu \mathrm{A}$.

L'électronique de traitement qui effectue cette mesure de courant est décrite en détail par CURTELIN [2]. Sa bande passante est de $580 \mathrm{~Hz}$.

\subsection{Etalonnage}

A géométrie fixée, la capacité mesurée $C_{m}$ est une fonction des variables suivantes:

$$
C_{m}=f\left(\varepsilon_{L}(T), \varepsilon_{G}(T), \varepsilon_{s}(T), \alpha, \text { configuration }\right)
$$

en supposant que le mélange en écoulement et que l'isolant sont à la même température $T$.

L'étalonnage in situ des trois voies du densitomètre en propane diphasique, en simulant divers taux de vide, n'est pas réalisable. Il est seulement possible de connaître la réponse de l'appareil plein (après remplissage en propane du réservoir de décompression, avant décompression) et vide (après décompression).

Pour vérifier les performances attendues de l'appareil, on simule hors section d'essais quelques taux de vide statiques par introduction de barreaux en Téflon. Quatre barreaux pleins cylindriques usinés aux diamètres $6,8,10$ et $12 \mathrm{~mm}$ simulent pour la configuration annulaire inverse (cœur liquide entouré d'un anneau gazeux) des taux de vide $\alpha=0,306,0,556,0,75$ et 1 respectivement. Un barreau creux de diamètres externe $12 \mathrm{~mm}$ et interne $8 \mathrm{~mm}$ simule pour la configuration annulaire directe (cœur gazeux entouré d'un anneau liquide) un taux de vide de 0,444 . Les mesures expérimentales sont comparées aux valeurs numériques issues de la résolution par éléments finis de l'équation de LAPLACE pour le potentiel dans une géométrie bidimensionnelle et avec des conditions aux limites représentatives. Afin d'analyser les résultats, considérons la capacité sous la forme adimensionnelle suivante :

$$
C_{\text {configuration }}^{*}(\alpha)=\frac{C(\alpha, \text { configuration })-C(1)}{C(0)-C(1)}
$$

où $C$ est déduite de la fonction $C_{m}$ de l'équation (6) en prenant $\varepsilon_{L}=\varepsilon_{s}=2,1$ et $\varepsilon_{G}=1$. Remarquons que pour $\alpha=0$ et $\alpha=1$, la notion de configuration disparaît.

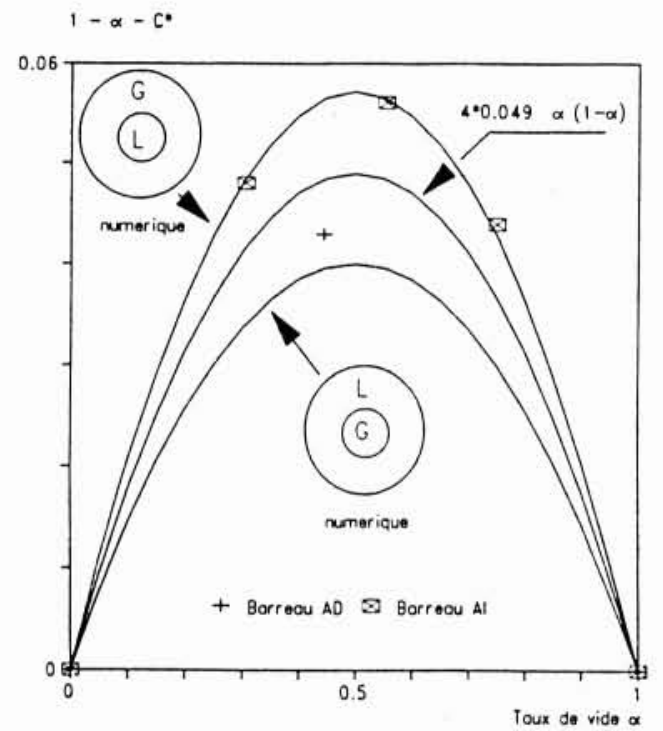

7. Etalonnage du densitomètre.

Comme $C^{*}(\alpha)$ ne montre qu'une très faible déviation par rapport à la relation affine $\alpha \rightarrow 1-\alpha$, on a tracé l'évolution (en fonction du taux de vide) de l'écart à la linéarité $1-\alpha-C^{*}$ pour les deux configurations extrêmes, ainsi que les valeurs issues des mesures avec les barreaux de Téflon (fig. 7). On constate que :

- la non-linéarité de l'appareil est faible, l'écart maximum absolu vaut 0,06 et est obtenu pour $\alpha=0,5$ en configuration annulaire inverse,

- l'incertitude absolue de détermination de la capacité due au changement de configuration est faible, et vaut au maximum 0,009 pour $\alpha=0,5$,

- l'accord entre les courbes déduites du calcul numérique et les "points d'étalonnage " en Téflon est excellent.

La meilleure approximation de la non-linéarité est la parabole située à égale distance des deux courbes représentant la non-linéarité de chaque configuration (fig. 7). Pour le Téflon, on trouve :

$$
1-\alpha-C^{*}=4(0,049 \pm 0,009) \alpha(1-\alpha) \text {. }
$$



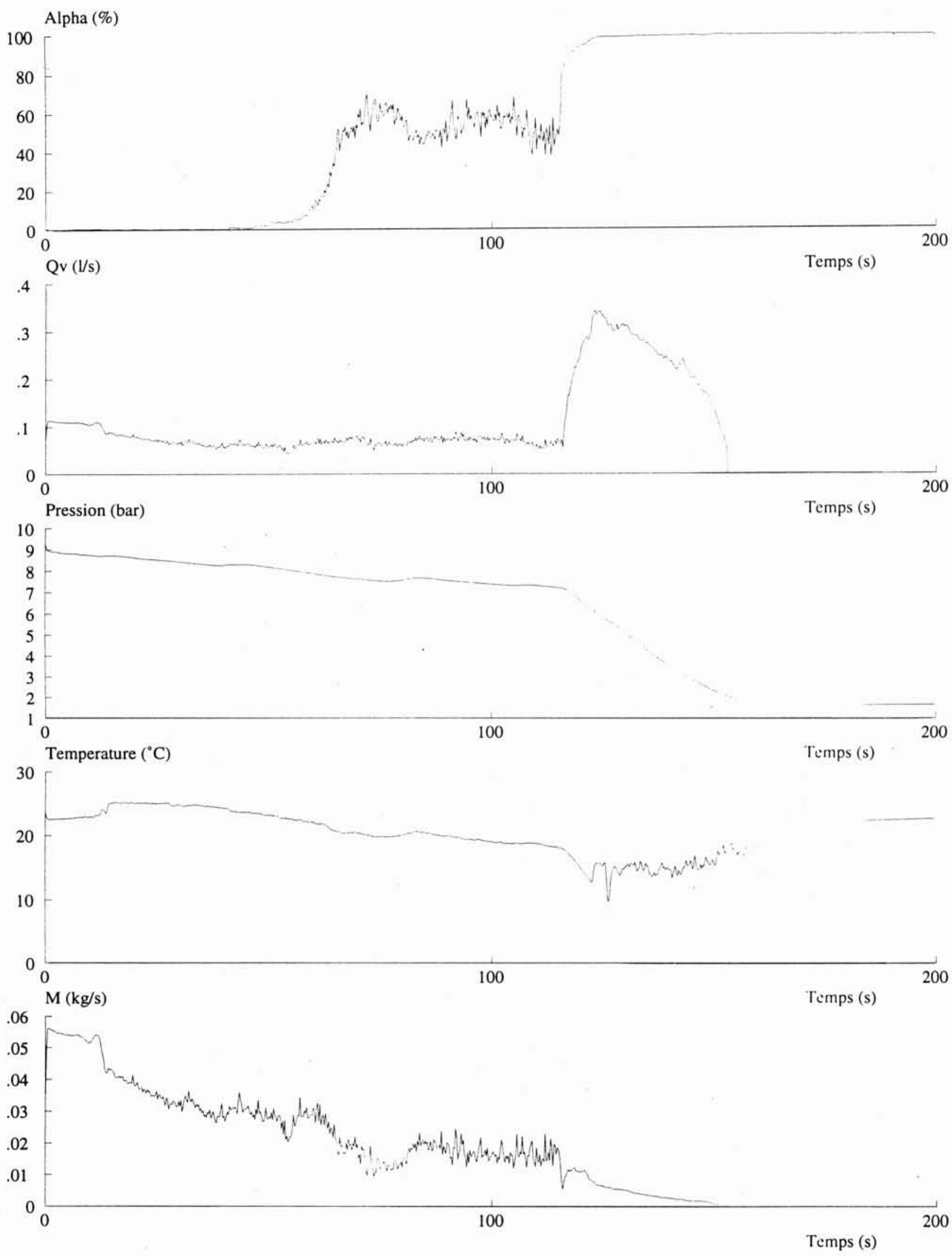

8. Décompression de 7,5 litres de propane à $25^{\circ} \mathrm{C}$ par une brèche de $2 \mathrm{~mm}$. 
Le bon accord entre calcul numérique et expérience en statique nous incite à utiliser, pour estimer la non-linéarité en propane, la courbe calculée de manière identique, mais avec les valeurs réelles de permittivité diélectrique du propane liquide et vapeur. Nous obtenons :

$$
1-\alpha-C^{*}=4(0,034 \pm 0,002) \alpha(1-\alpha) \text {. }
$$

Nous constatons qu'en propane, l'incertitude due à la configuration est 5 fois plus faible qu'avec le Téflon (qui s'explique par le plus faible contraste de permittivité). Cette incertitude serait donc, en pratique, négligeable devant les autres incertitudes.

\section{Mise en œuvre}

Nous présentons les signaux échantillonnés au cours de la vidange 7.5 litres de propane à $25^{\circ} \mathrm{C}$ au travers d'un orifice de $2 \mathrm{~mm}$ de diamètre. La figure 8 indique l'évolution temporelle du taux de vide $(8 a)$, du débit volumique $(8 b)$, de la pression $(8 c)$, de la température $(8 d)$ et du débit massique $(8 e)$.

Le taux de vide révèle que la décompression a lieu en trois étapes. La première, d'une durée de 50 secondes environ, indique un écoulement monophasique liquide. Le réservoir est tout simplement en train de se vider par le bas. La présence d'une stratification thermique initiale dans ce réservoir laisse apparaître un saut de température à 15 secondes du début, qui se traduit par une diminution brutale du débit massique. La seconde étape (de l'instant $t=50$ à $t=110$ secondes) montre un écoulement diphasique, avec un taux de vide fluctuant fortement autour $50 \%$. La visualisation a mis en évidence une forte génération de vapeur au niveau du fond du réservoir, due au destockage de chaleur de cette paroi. Une partie des bulles ainsi créées sont entraînées dans la ligne de décharge. Cet entraînement est nettement intermittent, ce qui explique le fort niveau fluctuant du taux de vide, et donc du débit massique. Cette seconde étape est suivie (à l'instant $t=115$ secondes environ) d'une brutale augmentation du taux de vide. La totalité du liquide a alors été expulsée du réservoir, et on assiste à une simple détente de vapeur, accompagnée par une forte chute de la température. C'est la troisième étape, dont la contribution en débit massique est très faible. La décompression s'achève lorsque les pressions dans les réservoirs de décompression et d'expansion s'équilibrent, au bout de 153 secondes.

En dernier lieu, signalons qu'en totalisant la masse écoulée par intégration temporelle du débit massique, on retrouve à mieux que $5 \%$ près la masse initiale avec laquelle le réservoir avait été rempli.

\section{Conclusion}

Une instrumentation originale a été développée afin de mesurer le débit massique d'un écoulement diphasique transitoire de propane, entre 0 et $60^{\circ} \mathrm{C}$. Cette mesure est réalisée grâce à la combinaison de capteurs de pression, dẻ température, de taux de vide et de débit volumique. La conception optimisée et l'étalonnage de chaque organe a pu garantir la qualité des résultats, et la mise en œuvre finale a permis de clairement identifier les différents mécanismes physiques régissant ce type d'écoulement.

\section{Remerciements}

Ce travail a été réalisé dans le cadre d'un programme de recherche groupée concernant les Risques Technologiques Majeurs, supporté par l'Institut de Protection et de Sûreté Nucléaire du CEA et par la Commission des Communautés Européennes (contrat MTH AA EV4T 00 19-F).

\section{Références}

[1] R. CURTEl. IN \& Ph. Vernier, 1990. - Behaviour of a flashing liquid within a vessel following loss of containment, Heat Transfer and Major Technological Hazards, Volume I, Eurotherm Seminar No 14, Brussels, May 15-17, Commission of the European Communities.

[2] R. CuRTElin, 1991. - Décompression d'un réservoir de propane liquéfié. Thèse de doctorat de l'Ecole Centrale de Paris (Spécialité : Transferts Thermiques).

[3] E. HeRVIEU, 1991. - Behavior of a flashing liquid within a vessel following loss of containment. Application to propane, Final Report, CEC Program : Major Technological Hazards.

[4] E. HeRVIEU, 1992. - Behavior of a flashing liquid within a vessel following loss of containment : application to propane, ANS proceedings, 1992 National Heat Transfer Conference, ANS, 227-225.

[5] G.F. POPPER, 1961. - In-core instrumentation for the measurement of hydrodynamic parameters in water-cooled reactors. Advanced course in in-core instrumentation for water-cooled reactors at Institut for Atomenergi, Kjeller, Norway, Vol. 1, Section V.IV.B, 32-39.

[6] G.F. Popper, 1961. - Proceedings of the power reactor in-core instrumentation meeting. Washington D.C., April 2829, TID 7598, Instruments Reactor Technology, 37-47.

[7] Z. ROUHANI, 1964. - Application of the turbine-type flowmeters in the measurement of steam quality and void. Symposium on in-core instrumentation, Oslo, June 1964, USAECCONF-640607.

[8] R. Frank, J. Mazars \& R. Ricque, 1977. - Determination of mass flowrate and quality using a turbine meter and a venturi, Heat and Fluid Flow in Water Reactor Safety. The Institution of Mech. Engrs., London, 63-68.

[9] H.M. HOCHREITER, 1958. - Dimensionless correlation of coefficients of turbine-type flowmeters, Trans. ASME, October, 1363-1368. 